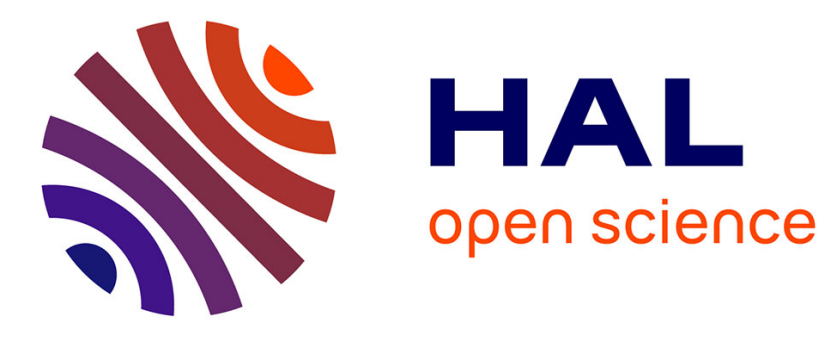

\title{
Micro-resonators based on integrated polymer technology for optical sensing
}

Pauline Girault, Jonathan Lemaitre, Mohammed Guendouz, Nathalie Lorrain, Luiz Poffo, Michel Gadonna, Dominique Bosc

\section{- To cite this version:}

Pauline Girault, Jonathan Lemaitre, Mohammed Guendouz, Nathalie Lorrain, Luiz Poffo, et al.. Micro-resonators based on integrated polymer technology for optical sensing. SPIE Photonics Europe 2014, Apr 2014, Brussels, Belgium. pp.914121, 10.1117/12.2052622 . hal-01017966

\section{HAL Id: hal-01017966 https://hal.science/hal-01017966}

Submitted on 3 Jul 2014

HAL is a multi-disciplinary open access archive for the deposit and dissemination of scientific research documents, whether they are published or not. The documents may come from teaching and research institutions in France or abroad, or from public or private research centers.
L'archive ouverte pluridisciplinaire $\mathbf{H A L}$, est destinée au dépôt et à la diffusion de documents scientifiques de niveau recherche, publiés ou non, émanant des établissements d'enseignement et de recherche français ou étrangers, des laboratoires publics ou privés. 


\title{
Micro-resonators based on integrated polymer technology for optical sensing.
}

\author{
Pauline Girault, Jonathan Lemaitre, Mohammed Guendouz*, Nathalie Lorrain, Luiz Poffo, \\ Michel Gadonna and Dominique Bosc \\ Université européenne de Bretagne \\ CNRS, Laboratoire FOTON, UMR 6082, ENSSAT, BP 80518, 22305 Lannion Cedex, France \\ *mohammed.guendouz@univ-rennes1.fr
}

\begin{abstract}
Research on sensors has experienced a noticeable development over the last decades especially in label free optical biosensors. However, compact sensors without markers for rapid, reliable and inexpensive detection of various substances induces a significant research of new technological solutions. The context of this work is the development of a sensor based on easily integrated and inexpensive micro-resonator (MR) component in integrated optics, highly sensitive and selective mainly in the areas of health and food.

In this work, we take advantage of our previous studies on filters based on micro-resonators (MR) to experiment a new couple of polymers in the objective to use MR as a sensing function. MRs have been fabricated by processing SU8 polymer as core and PMATRIFE polymer as cladding layer of the waveguide. The refractive index contrast reaches $0.16 @ 1550 \mathrm{~nm}$. Sub-micronic ring waveguides gaps from 0.5 to $1 \mu \mathrm{m}$ have been successfully achieved with UV (i-line) photolithography. This work confirms our forecasts, published earlier, about the resolution that can be achieved. First results show a good extinction coefficient of $\sim 17 \mathrm{~dB}$, a quality factor around $10^{4}$ and a finesse of 12 . These results are in concordance with the theoretical study and they allow us to validate our technology with this couple of polymers. Work is going on with others lower cladding materials that will be used to further increase refractive index contrast for sensing applications.
\end{abstract}

Keywords: micro-resonators, polymers, integrated optics, photolithography

\section{INTRODUCTION}

Over the last decades, the growing need for compact sensors and without markers, that allow rapid and inexpensive reliable detection of various substances, induces a significant research of new technological solutions [1].

In this context, the aim of this work is to improve optical sensors based on easily integrated and inexpensive micro-resonator (MR) component. Indeed, MRs can act as highly sensitive and selective functions mainly in the areas of health and food [2]. MRs offer the advantage of significantly reducing the device size and can be a key element of a labon-chip sensor system $[3,4]$.

This study takes place in the field of integrated optics based on polymer technology. Although low quality factor [5], these materials are largely used for MRs in integrated optics thanks to their low cost and their easy manufacturing process [6]. Moreover, their compatibility with microfluidic and their capability to be functionalized through surface modification methods, in order to attach biological sites, make them advantageous materials for sensing applications [7].

Taking advantage of our previous experiments on filters based on MRs [8-10], we present in this paper a study of a MR using a new couple of polymers and sub-micronic ring waveguides gaps. SU8 polymer as core and PMATRIFE polymer as cladding layer have been chosen regarding their refractive index contrast (0.16@1550 nm) and sensing applications. This work provides the first results that will be carried on with the sensing study. After theoretical reminders considered in the calculations of MRs characteristics, we present first experimental measures of extinction and 
extraction responses that are encouraging for the sensing study. Coupling and losses parameters are deduced from theoretical adjustments. Optical results are discussed taking into account SEM observations and previous results [8-10].

\section{DESIGN AND FABRICATION}

\subsection{Principle}

Double side coupled micro-ring resonator consists of two straight waveguides; the first one is used for signal injection and the second one is used for signal extraction (figure 1). It operates as a Fabry-Perot interferometer; the wavelengths injected ( $\Sigma \lambda$, from broadband source) through the first waveguide are coupled into the ring. Those, which are in tune with the incident wavelengths after round trips in the ring, resonate by constructive interferences. The spectral analyze, out of the injection straight waveguide, shows an extinction for each resonant wavelength (transmitted signal) while, out of the second straight waveguide (dropped signal), the spectral response shows the extracted resonant wavelengths.

In the coupling region, we define:

- $\quad \tau$ and $\tau$, the amplitude self-coupling coefficients in the straight waveguide and in the ring waveguide respectively and,

- $\quad \kappa$ and $\kappa$ ', the amplitude cross-coupling coefficients from the straight waveguide to the ring waveguide and from the ring waveguide to the straight waveguide respectively.

- $\quad$ In case of symmetrical coupling, $\tau=\tau^{\prime}, \kappa=\kappa^{\prime}$.

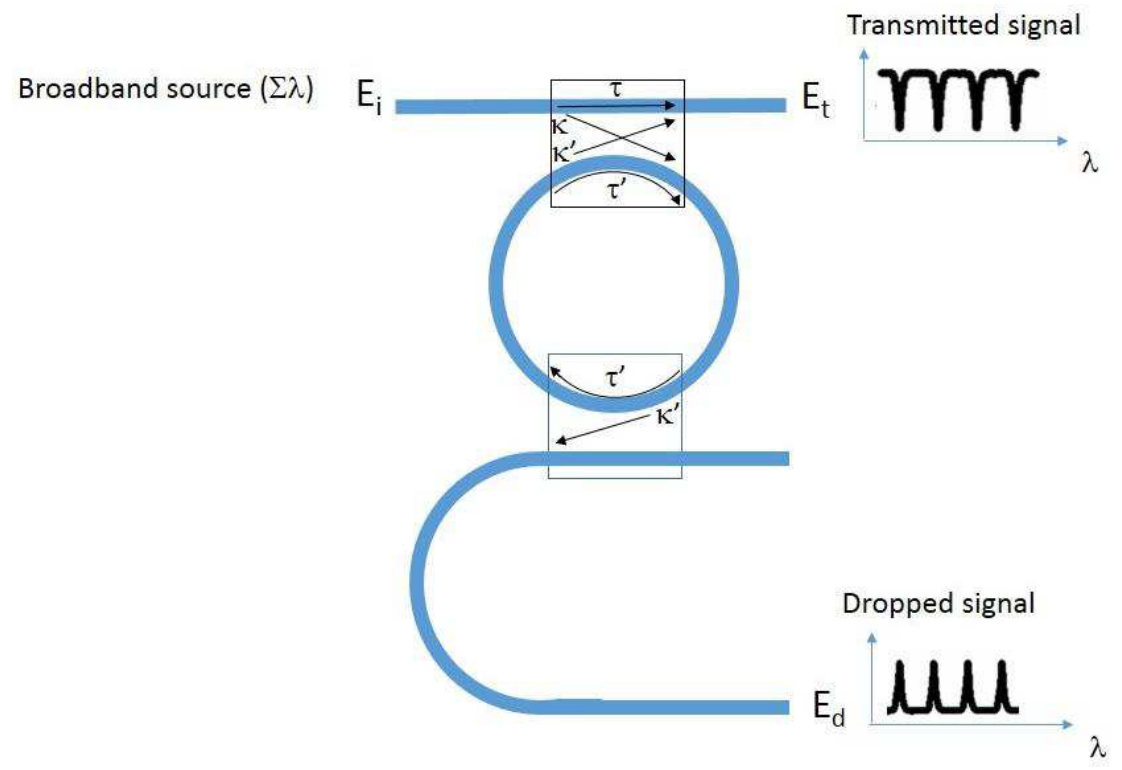

Figure 1. Double side coupled micro-ring resonator. The field amplitudes are denoted by $\mathrm{E}_{\mathrm{i}}$ (incident), $\mathrm{E}_{\mathrm{t}}$ (transmitted) and $E_{d}$ (dropped). Self-coupling ( $\tau$ and $\left.\tau^{\prime}\right)$ and cross-coupling ( $\kappa$ and $\left.\kappa^{\prime}\right)$ amplitude coefficients are indicated.

Using the notation in figure 1 and taking into account a symmetrical coupling, the normalized transmitted intensity and the normalized dropped intensity can be expressed by [11]:

$$
T(\lambda)=\left|\frac{E_{t}}{E_{i}}\right|^{2}=\eta^{2}\left|\frac{\tau-\eta^{2} a \tau \exp (j \phi)}{1-\eta^{2} a \tau^{2} \exp (j \phi)}\right|^{2} \text { and } D(\lambda)=\left|\frac{E_{d}}{E_{i}}\right|^{2}=\eta^{2}\left|\frac{\sqrt{a} \kappa^{2} \exp (j \phi / 2)}{1-\eta^{2} a \tau^{2} \exp (j \phi)}\right|^{2}
$$

where:

$-E_{i}, E_{t}$ and $E_{d}$ are respectively, the amplitude of the incident, transmitted and dropped fields, 
- $\phi$ is the single-pass phase shift of the propagated wave in the ring, $\phi=2 \pi R n_{e f f} \beta$, with $\beta$, the propagation constant, $\mathrm{n}_{\text {eff }}$, the effective refractive index of the ring waveguide and $\mathrm{R}$, the ring radius.

- a is the field attenuation constant such as $a=\exp (-2 \pi R \alpha)$, and $\alpha$ is the propagation losses per length unit,

- $\eta$ is the coupling losses coefficient; in case of lossless coupling, $|\tau|^{2}+|\kappa|^{2}=1$.

Two consecutive wavelengths extracted from the ring are separated by a Free Spectral Range (FSR) such as: $F S R=\frac{\lambda^{2}}{2 \pi R n_{g}}$, where $\mathrm{n}_{\mathrm{g}}$ is the group refractive index such as $n_{g}(\lambda)=n_{\text {eff }}(\lambda)-\lambda \frac{d n_{\text {eff }}}{d \lambda}$.

Quality factor $(Q=\lambda / \delta \lambda)$, Finesse $(F=F S R / \delta \lambda)$, Extinction $\left(T_{\max }-T_{\min }\right)$ or/and Extraction $\left(D_{\max }-D_{\min }\right)$ efficiencies are the characteristic values of MRs which depend, for a given design, on the coupling ratio between ring and straight waveguides and on the round trip losses.

\subsection{Design and implementation}

In this paper, we report the fabrication of micro-ring resonators with Epoxy photoresist SU8 core material as compared to our previous work for which core was made in PVCi [9]. SU8 has been chosen for its optical properties and its physico-chemical and biological compatibilities, properties for which SU8 is often used for optical sensing applications; microfluidic and surface functionalizations can be easily performed [12]. PMATRIFE (Poly(2,2,2 MethAcrylate of TRIFluoro-Ethyle)) polymer $(\mathrm{n}=1.405 @ 1.55 \mu \mathrm{m})$ has been chosen for lower and upper cladding layers. Refractive index @ $1.55 \mu \mathrm{m}$ of PVCi $(\mathrm{n}=1.58)$ and SU8 $(\mathrm{n}=1.57)$ being close, we have used the same design as in our previous study [9-10]. The top view of the design and the corresponding cross section are presented on figure 2 . The ring and straight waveguides are made of the same materials. To provide single mode propagation and high confinement, designed height and width of the waveguide core are $1.5 \mu \mathrm{m}$ x $1.5 \mu \mathrm{m}$. The gap separation $\mathrm{g}$ between the straight waveguide and the ring is an important parameter which contributes to the coupling coefficient. Regarding to the theoretical photolithography resolution limit of $0.4 \mu \mathrm{m}$, gaps varying from 0.5 to $1 \mu \mathrm{m}$ have been studied [13]. Radius ring of $120 \mu \mathrm{m}$ used in the design provides minimum bend losses regarding the effective refractive index of the waveguide (around 1.49). The expected FSR of $2 \mathrm{~nm}$ will allow us to explore a large detection window. In figure 3, we describe the realization process.

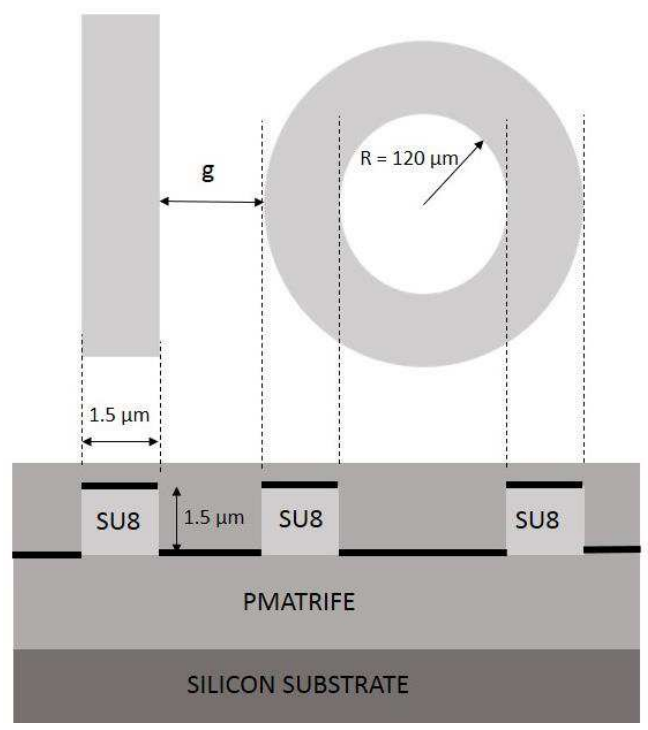

Figure 2. Scheme of a top view and a cross section of the micro-ring resonator. 
In class 100 clean-room, all the polymer solutions used during the process (figure 3) were filtered at $0.2 \mu \mathrm{m}$ before the spin-coating steps. A $7 \mu \mathrm{m}$-thick layer of PMATRIFE (500g/l in THF solvent) was deposited by spin coating on $200 \mu \mathrm{m}$-thickness silicon substrate. The deposited layer is submitted to oxygen plasma RIE (Reactive Ion Etching) to promote the SU8 adherence. A $1.5 \mu$ m-thick layer of SU8 is spin-coated and submitted to a thermal treatment up to $95^{\circ} \mathrm{C}$. Over the SU8 core, a thin layer of silica $(\sim 25 \mathrm{~nm})$ and then positive photosensitive resin $(\sim 400 \mathrm{~nm})$ are deposited. The thin $\mathrm{SiO}_{2}$ layer allows to protect $\mathrm{SU} 8$ from the next UV exposure step. Then, patterns were produced through a chrome photolithography mask under UV exposure and developed. To obtain the desired waveguides, an oxygen plasma RIE was performed. A thin layer of silicon nitride $(\sim 35 \mathrm{~nm})$ was deposited to prevent the dissolution of the under cladding layer by the solvent of the upper cladding. Finally, the PMATRIFE upper cladding layer $(6 \mu \mathrm{m})$ was deposited, also by spin-coating, and submitted to a thermal treatment up to $80^{\circ} \mathrm{C}$.

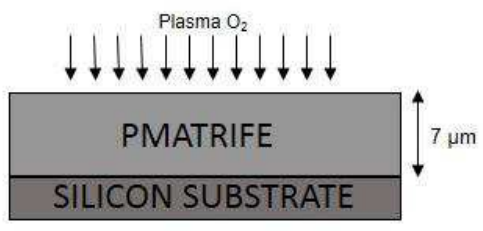

(a)

\begin{tabular}{|c|c|c|}
\hline SU8 & SU8 & SU8 \\
\hline \multicolumn{3}{|c|}{ PMATRIFE } \\
\hline SI & ON SI & $\overline{A T E}$ \\
\hline
\end{tabular}

(d)

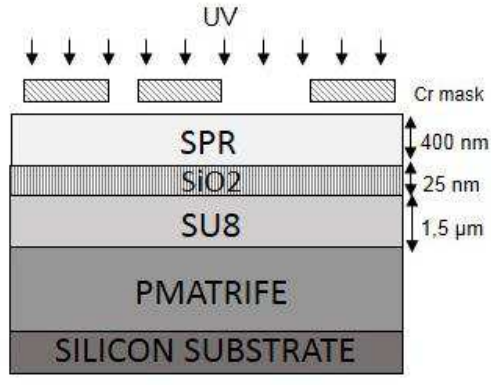

(b)

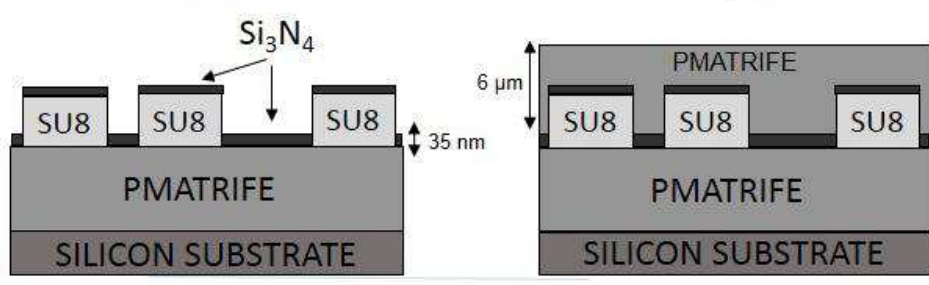

(e)

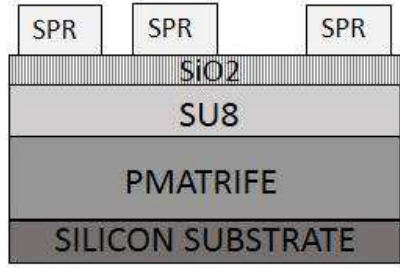

(c)

(f)

Figure 3. Fabrication process for the micro-ring resonator. Scheme of the photolithography process used for the fabrication of micro-ring resonator polymer: deposit of a) the PMATRIFE lower cladding by spin coating; b) the SU8 core, the $\mathrm{SiO}_{2}$ protect layer, the SPR photosensitive resin and irradiation under UV exposure; c) wet etching development of the photosensitive resin; d) dry etching of the $\mathrm{SiO}_{2}$ protect layer and the $\mathrm{SU} 8$ core polymer; deposit of e) the $\mathrm{Si}_{3} \mathrm{~N}_{4}$ protect layer and f) the PMATRIFE upper cladding.

\subsection{Characterizations}

In order to characterize MRs, we have measured the spectral responses according to different gaps. A tunable wavelength laser was injected in the input waveguide using a lensed fiber with a mode radius of $1.3 \mu \mathrm{m}$. At the waveguide output, a lensed single mode fiber is also used to couple the output of the waveguide to a power meter or to the optical spectrum analyzer. Polarization controller's is inserted between the output of the lensed fiber and the detector. The measurements have been done at the through port $\left(E_{t}\right)$ and at the drop port $\left(E_{d}\right)$ in the wavelength range of 1550$1560 \mathrm{~nm}$ with a step resolution of $2.5 \mathrm{pm}$. In order to resolve more precisely resonant peak and bandwidth, measurements with a step of 1 pm have been performed. Scanning Electronic Microscopy (SEM) observations have been also performed on the top and on the cross section in order to appreciate the quality of the etching edges and to verify the structure dimensions.

\section{RESULTS AND DISCUSSIONS}

SEM top view images (figure 4) after the step (d) presented in figure 3, show the whole micro-ring structure (figure $4 \mathrm{a}$ ) and the coupling region (figure $4 \mathrm{~b}$ and $4 \mathrm{c})$. The intern ring radius is $(120 \pm 2) \mu \mathrm{m}$, the waveguide width is 
$(1.5 \pm 0.1) \mu \mathrm{m}$ and the gap between the straight waveguides and the ring is $(0.7 \pm 0.1) \mu \mathrm{m}$. Figure $4 \mathrm{~d}$ shows a tilted view of a straight waveguide at a higher magnification to observe the etching edges which are quite satisfying at this scale. However, an appreciation of the roughness is difficult to obtain.
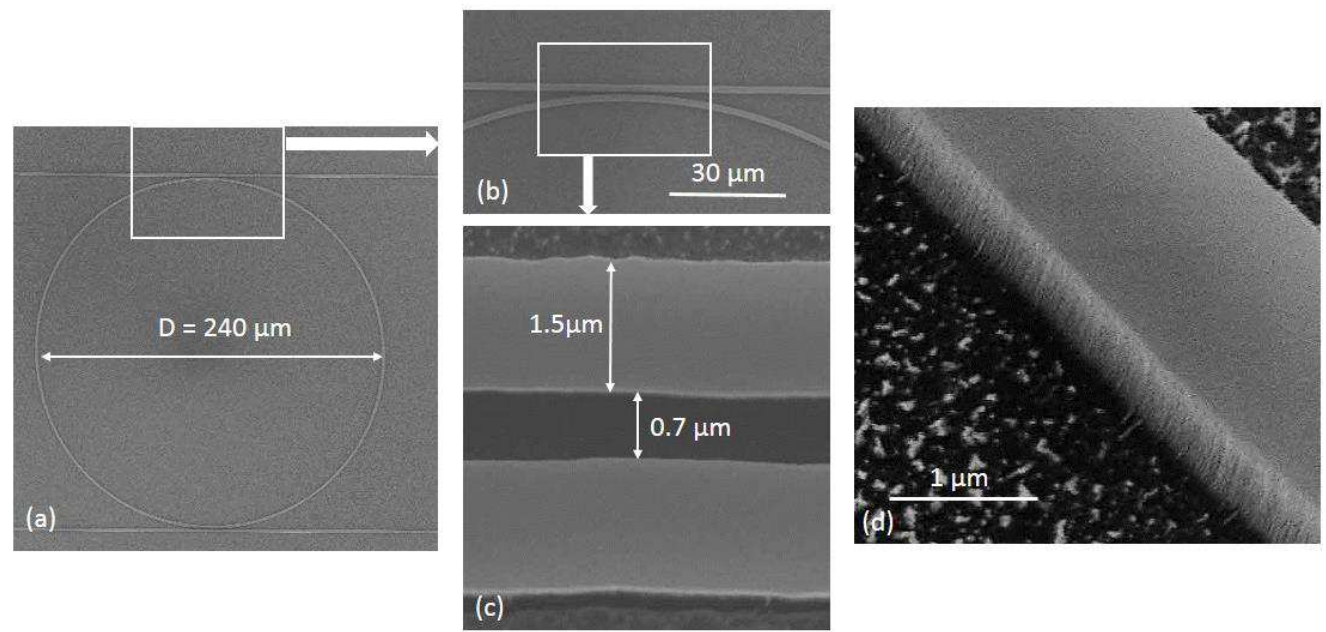

Figure 4: SEM images of the MR (a), the coupling region (b, c) and the edges of the SU8 core waveguide (d).

SEM cross section views (figure 5) show the SU8 core (width: $1.4 \pm 0.2 \mu \mathrm{m}$; thickness: $1.4 \pm 0.1 \mu \mathrm{m}$ ) surrounded by a $7 \mu \mathrm{m}$ and $6 \mu \mathrm{m}$ thick-cladding lower and upper layers of PMATRIFE respectively (figure 5a). High roughness appears in the cladding cross-section which is due to a cleavage effect. The view at higher magnification (figure $5 \mathrm{~b}$ ) reveals the core waveguide with the expected dimensions and a quite good cleavage surface. We can also distinguish an upper layer which can be attributed to nitride that has been used to prevent the dissolution of the lower PMATRIFE layer. One can observe a detachment of the upper layer on the top of SU8 waveguide which is attributed to the cleavage. This effect is neglected for optical propagation modelization.
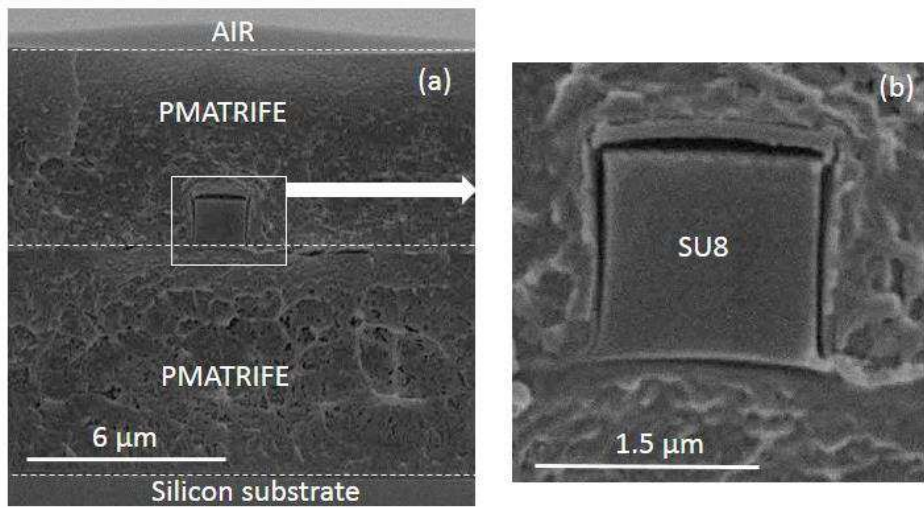

Figure 5: SEM cross-section images of the PMATRIFE/SU8/PMATRIFE waveguide: (a) whole view and (b) at higher magnification.

The spectral responses of fabricated micro-ring resonator with different gaps (from 0.5 to $1 \mu \mathrm{m}$ ) were measured and compared to the calculated ones according to the formulas reminded on section 2.1. Adjustment of the theoretical and experimental spectra allows us to deduce especially the coupling ratio and the propagation losses in the ring. The best extinction efficiency was obtained for a gap of $0.7 \mu \mathrm{m}$. Figure 6 shows the transmitted and dropped spectra of this microring resonator for one polarization light. We can observe background periodic oscillations which are due to reflection effect of cleaved polymer facets (FSR of $0.1 \mathrm{~nm}$ corresponding to an interferometric length of $0.75 \mathrm{~cm}$ ). From the experimental spectra, we have deduced the following characteristics of the resonator: an extinction rate around 
$17 \mathrm{~dB}$ and an extraction rate of $16 \mathrm{~dB}$. A FSR of $2 \mathrm{~nm}$ has been deduced and corresponds to the calculated one. A full width at half maximum $(\delta \lambda)$ of $0.165 \mathrm{~nm}$, with a corresponding quality factor Q of 9416 and a finesse $\mathrm{F}$ of 12 have been obtained.

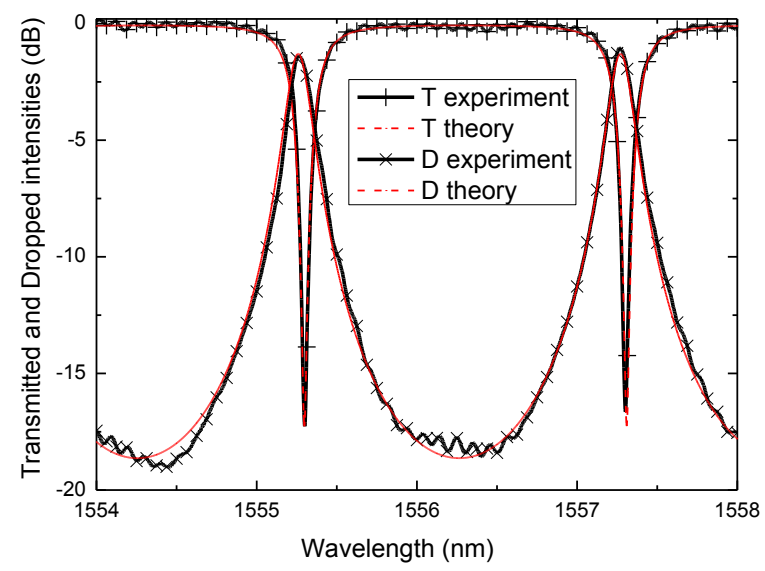

Figure 6: Experimental and calculated Transmission and Drop spectra for one polarization light.

A good agreement between experimental and calculated spectra is obtained for the following parameters: coupling coefficient $\mathrm{k}^{2}=0.21, \eta=1$ (no coupling losses), field attenuation constant $\mathrm{a}=0.9631$ which corresponds to propagation losses per length unit $(\alpha)$ of $2.15 \mathrm{~dB} / \mathrm{cm}$ and group refractive index $n_{g}=1.587$. From the calculated effective refractive index of 1.49 , we have deduce the wavelength dispersion effect $\left(\mathrm{dn}_{\mathrm{eff}} / \mathrm{d} \lambda\right)$ around $-6.10^{-6} \mathrm{~nm}^{-1}$. The propagation losses per length unit value around $2 \mathrm{~dB} / \mathrm{cm}$ is in a good agreement with those cited in literature with single straight SU8 waveguides [14] and is lower than others $(5 \mathrm{~dB} / \mathrm{cm})$ obtained in micro-ring resonators using SU8 for core but OG-125 $(n=1.456)$ for lower and upper cladding layers [15]. The quality of etching edges that has been observed on SEM images can explain these lower losses obtained with our technology.

Figure 7 represents the extinction and extraction efficiency in function of the coupling coefficient $\kappa^{2}$. This last coefficient around $20 \%$ corresponds to the maximal extinction efficiency while for the drop case, higher $\kappa^{2}$ should be achieved to obtain a better extraction efficiency.

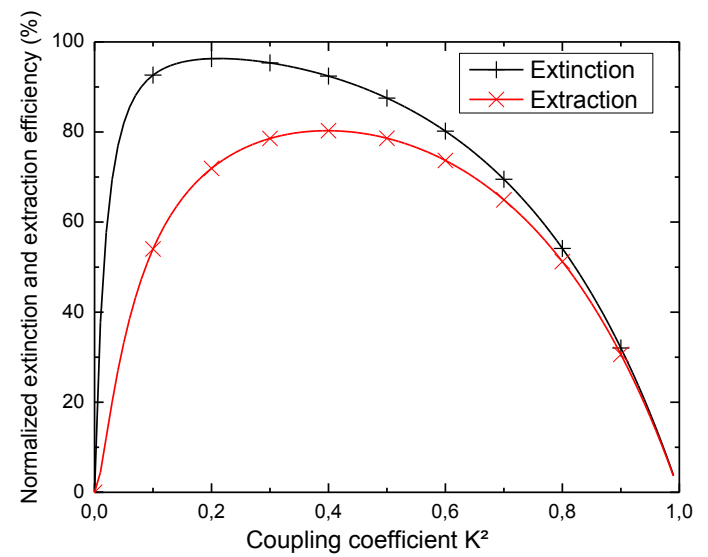

Figure 7: Normalized extinction and extraction efficiency as a function of the coupling coefficient $\mathrm{k}^{2}$. 
Compared to our previous results using PVCi for core material, a coupling rate of $23 \%$ was obtained for a lateral gap of $0.8 \mu \mathrm{m}$. Better quality factor $\mathrm{Q}$ and finesse $\mathrm{F}$ were obtained $\left(\mathrm{Q}=1.210^{4}\right.$ and $\left.\mathrm{F}=17\right)$. Also, higher extinction efficiency of $20 \mathrm{~dB}$ was obtained. Lower propagation losses in PVCi $(1 \mathrm{~dB} / \mathrm{cm} @ 1.55 \mu \mathrm{m})$ [16] can explained these differences in the results. Moreover, another explanation of these MR performances could be due to the presence of the nitride layer which could induce leaked modes in the cladding. Nevertheless, these results are promising and further work will consist in performing micro-rings without upper cladding. Moreover, we will investigate others lower cladding materials for sensing applications.

\section{CONCLUSIONS AND PERSPECTIVES}

This study aimed to demonstrate the fabrication feasibility, with our technology previously used for wavelength tunable filters, of integrated MRs using SU8 polymer for sensing applications. Indeed, the SU8 is a biocompatible polymer which is largely used in integrated optical sensors. PMATRIFE polymer has been used for lower and upper cladding layers. Technology has been successfully adapted for this couple of polymers to fabricate MRs with sub-micronic gaps varying from 0.5 to $1 \mu \mathrm{m}$. The best extinction efficiency $(17 \mathrm{~dB})$ with a quality factor of nearly $10^{4}$ has been achieved for a gap of $0.7 \mu \mathrm{m}$ corresponding to a coupling rate of $21 \%$ according to the theoretical MR responses. Work is going on for sensing applications using a micro-ring without the PMATRIFE upper cladding that will be replaced by the sensing medium. Detection of glucose content in water will be then performed (PMATRIFE / SU8 /sensing medium) in order to characterize micro-rings. For the estimated effective refractive index of 1.48 , a coupling rate around $20 \%$ is suitable to obtain a sufficient extinction efficiency. To reach such a value, resonators with a lower gap, around $0.5 \mu \mathrm{m}$, should be studied. Using the previously described technology, we already have demonstrated that such a gap could be achieved. Estimation of the sensor sensitivity with this couple of polymers approaches $100 \mathrm{~nm} / \mathrm{RIU}$ (Refractive Index Unit), value which is of the same order than those published in literature [17]. In order to improve the sensitivity, others lower cladding materials, with low refractive index, will be studied.

\section{ACKNOWLEDGMENTS}

This work is supported by the PONANT project, funded by Lannion Trégor Communauté, Conseil Général des Côtes d'Armor, Région Bretagne, Feder and the French Research Ministry.

\section{REFERENCES}

[1] Fan, X., White, I. M., Shopova, S. I., Zhu, H., Suter, J. D., Sun, Y., “Sensitive optical biosensors for unlabeled targets: A review," Analytica Chimica Acta, 620, 8-26 (2008).

[2] Vollmer, F., Arnold, S., "Whispering-gallery-mode biosensing: label-free detection down to single molecules," Nature Methods, vol.5, n7, 591-596 (2008).

[3] De Vos, K., Girones, J., Popelka, S., Schacht, E., Baets, R., Bienstman, P., "SOI optical micro-ring resonator with poly(ethylene glycol) polymer brush for label-free biosensor applications," Biosensors and Bioelectronics, 24, 25282533 (2009).

[4] Chao, C. Y., Guo, L. J., "Design and optimization of micro-ring resonators in biochemical sensing applications" Journal of Lightwave Technology”, vol.24, n³, 1395-1402 (2006).

[5] Arnold, S., Khoshsima, M., Teraoka, I., Holler, S., Vollmer, F., "Shift of whispering-gallery modes in microspheres by protein adsorption," Optics Letters, 28, 272-274 (2003).

[6] Kim, G. D., Son, G. S., Lee, H. S., Kim, K. D., Lee, S. S., "Integrated photonic glucose biosensor using a vertically coupled micro-ring resonator in polymers," Optics Communications, 281, 4644-4647 (2008).

[7] Chao, C. Y., Guo, L. J., "Biochemical sensors based on polymer micro-rings with sharp asymmetrical resonance," Applied Physics Letters, vol.83, n 8, 1527-1529 (2003).

[8] Maalouf, A., Gadonna, M., Bosc, D., Lobo, S., Bramerie, L., "Study of polymer micro-ring resonator based filter in system experiment at $10 \mathrm{Gbit} / \mathrm{s}$, , ECIO'10 Proceedings, ref. WeP34 (2010).

[9] Maalouf, A., Gadonna, M., Bosc, D., Hardy, I., "Integrated polymers (PVCi/PMATRIFE) micro-ring resonators for low power tunable filters," Optics Communications, 285, 4088-4091 (2012). 
[10] Maalouf, A., Bastianelli, C., Mahé, F., Belmiloudi, A., Gadonna, M., Bosc, D., "Improved integrated resonators in polymer technology for tunable filter," Photonics Europe 2012, Silicon Photonics and Photonic Integrated Circuits conference, Brussels, SPIE Vol. 8431 - 75 (2012) .

[11] Yariv, A., "Universal relations for coupling of optical power between micro-resonators and dielectric waveguides," Electronics Letters, vol.36, n4, 321-322 (2000).

[12] Gadre, A. P., Nijdam, A. J., Garra, J. A., Monica, A. H., Cheng, M. C., Luo, C., Srivastava, Y. N., Schneider, T. W., Long, T. J., White, R. C., Paranjape, M., Currie, J. F., "Fabrication of a fluid encapsulated dermal patch using multilayered Su-8," Sensors and Actuators A, 114, 478-485 (2004).

[13] Maalouf, A., Gadonna, M., Bosc, D., "An improvement in standard photolithography resolution based on Kirchhoff diffraction studies," Journal of Physics D: Applied Physics, 42 (2009).

[14] Bêche, B., Pelletier, N., Gaviot, E., Zyss, J., "Single-mode TE00-TM00 optical waveguides on SU-8 polymer," Optics Communications, 230, 91-94 (2004).

[15] Poon, J. K. S., Huang, Y., Paloczi, G. T., Yariv, A., "Soft lithography replica molding of critically coupled polymer micro-ring resonators," IEEE Photonics technology letters, vol.16, n¹1, 2496-2498 (2004).

[16] Bosc, D., Grosso, P., Hardy, I., Assaïd, I., Batte, T., Haesaert, S., Vinouze, B., "High refractive index contrast in a photosensitive polymer and waveguide photo-print demonstration," Optics Communications, 235, 281-284 (2004).

[17] Ciminelli, C., Dell'Olio, D., Conteduca, D., Campanella, C.M., Armenise, M.N., "High performance SOI microring resonator for biochemical sensing," Optics \& Laser Technology, 59, 60-67 (2014). 